\title{
Feasibility of Design in Stereolithography
}

\author{
B. Asberg, G. Blanco, P. Bose, J. Garcia-Lopez, M. Overmars, \\ G. Toussaint, G. Wilfong, and B. Zhu
}

$\underline{\text { UU-CS-1994-25 }}$

June 1994

\section{Utrecht University}




\title{
Feasibility of Design in Stereolithography
}

\author{
B. Asberg, G. Blanco, P. Bose, J. Garcia-Lopez, M. Overmars, \\ G. Toussaint, G. Wilfong, and B. Zhu
}

Technical Report UU-CS-1994-25

June 1994

Department of Computer Science Utrecht University

P.O.Box 80.089 3508 TB Utrecht

The Netherlands 
ISSN: 0924-3275 


\title{
Feasibility of Design in Stereolithography
}

\author{
Boudewijn Asberg*, Gregoria Blanco, Prosenjit Bose, Jesus Garcia-Lopez ${ }^{\dagger}$ \\ Mark Overmars*, Godfried Toussaint $\ddagger$ Gordon Wilfong§, and Binhai Zhu ${ }^{\ddagger}$
}

\begin{abstract}
We study the feasibility of design for a layer-deposition manufacturing process called stereolithography which works by controlling a vertical laser beam which when targeted on a photocurable liquid causes the liquid to harden. In order to better understand the power as well as the limitations of this manufacturing process, we define a mathematical model of stereolithography (referred to as vertical stereolithography) and analyze the class of objects that can be constructed under the assumptions of the model. Given an object (modelled as a polygon or a polyhedron), we give algorithms that decide in $O(n)$ time whether or not the object can be constructed by vertical stereolithography. If the answer is in the affirmative, the algorithm reports a description of all the orientations in which the object can be made. We then define a more flexible model that more accurately reflects the actual capabilities of stereolithography (referred to as variable-angle stereolithography) and again study the class of feasible objects for this model. We give an $O(n)$ time algorithm for polygons and $O(n \log n)$ as well as $O(n)$ time algorithms for polyhedra. We show that objects formed with variable-angle stereolithography can also be constructed using another manufacturing process known as gravity casting. Furthermore, we show that the polyhedral objects formed by vertical stereolithography are closely related to polyhedral terrains which are important structures in geographic information systems (GIS) and computational geometry. In fact, an object built with variable-angle stereolithography resembles a terrain with overhangs, thus initiating the study of more realistic terrains than the standard ones considered in geographic information systems. Finally, we relate our results to the area of grasping in robotics by showing that the polygonal and polyhedral objects that can be built by vertical stereolithography can be clamped by parallel jaw grippers with any positive sized gripper.
\end{abstract}

\section{Introduction}

A CAD/CAM system allows an engineer to design a product using the CAD system, store a description of the product in a data base and from the description in the data base, derive a sequence of actions for the computer controlled machines (CCMs) in order to manufacture the product. The class of products that can be realized by a particular system is constrained by

\footnotetext{
${ }^{*}$ Dept. of Computer Science, Utrecht University, P.O. Box 80.089, 3508 TB, Utrecht, Netherlands. This research was partially supported by the ESPRIT Basic Research Action No. 7141 (project ALCOM II) and by the Netherlands Organization for Scientific Research (N.W.O.).

${ }^{\dagger}$ Escuela Universitaria de Informatica Madrid, Spain

${ }^{\ddagger}$ School of Computer Science, McGill University, 3480 University Street, Montreal, Quebec, Canada, H3A 2 A7. This research was supported in part by an NSERC-postgraduate scholarship, and grants NSERC-OGP0009293, FCAR-93ER0291

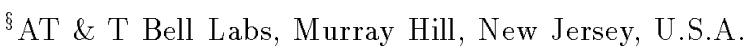




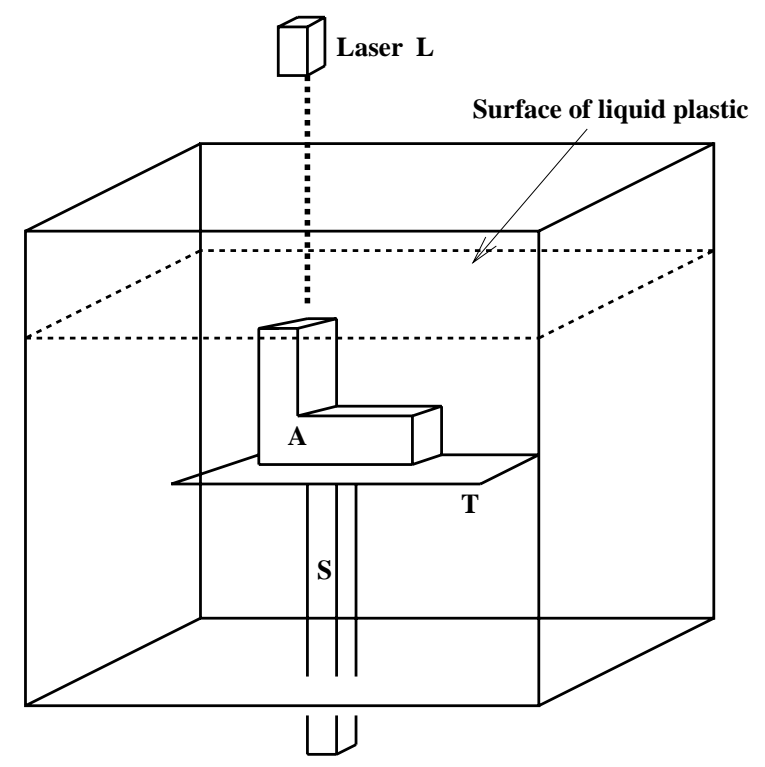

Figure 1: Stereolithograpy system.

the versatility of the CAD system and the number of each type of CCM available. Therefore a useful feature of such a system would be the ability to decide feasibility of manufacture, that is, it would be useful to be able to decide whether or not a particular design is realizable by an existing configuration of CCMs.

We consider the problem of deciding whether or not a design is feasible for a CAD/CAM system developed and patented by 3D Systems of Sylmar, CA that employs a process called stereolithography. (See Figure 1). The components of the stereolithography manufacturing process consist of a vat of liquid photocurable plastic, a computer controlled table $T$ on a stand $S$ that can be moved up and down in the vat and a laser $L$ above the vat that can shine on the surface of the liquid plastic and can move in a horizontal plane. The system works as follows. At the first step the table is just below the surface of the plastic and the laser is controlled to move about so that the light shines on the surface of the plastic and draws the bottom-most cross-section of the object $A$ being built. When the laser light contacts the plastic, the plastic solidifies and so the first cross-section of the object is formed and rests on the table. At the next step the table is lowered a small amount to allow liquid to cover the hardened layer and the laser then draws the next cross-section of the object. The light from the laser penetrates the liquid just deep enough so that this cross-section is welded to the lower cross-section produced at the previous step. This process is repeated until the entire object is formed. The direction given by a normal to the table pointing from the laser is called the direction of formation for the object.

There are some objects that can be formed only if the direction of formation is chosen correctly. For example, in Figure 2, the object (a) can not be formed in the position shown. Consider what occurs when the cross-section is reached where the surface $S$ lies. The surface $S$ is not supported below and so as it is formed it sinks to the level of the table. However, if the object is formed 


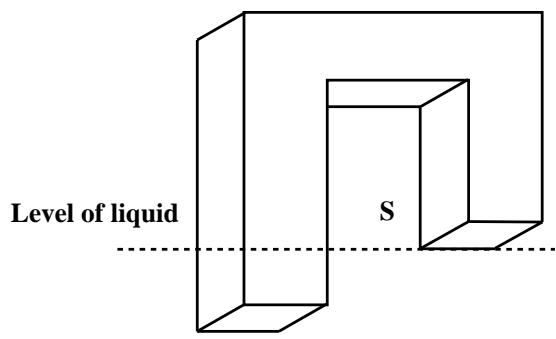

(a)

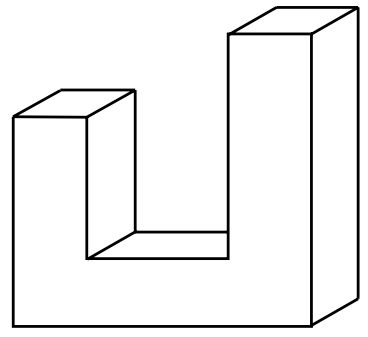

(b)

Figure 2: Infeasible and feasible directions of formation.

in the opposite direction as in Figure 2 object (b) then stereolithography will succeed. Naturally, there are some objects that can not be formed using stereolithography regardless of the direction of formation chosen.

In order to better understand the power as well as the limitations of this manufacturing process, we define a mathematical model of stereolithography (referred to as vertical stereolithography). Under this model, we assume that each layer can be welded on to the previous such that no part of the top layer hangs over the previous. We analyze the class of objects that can be constructed under the assumptions of the model. Given an object (modelled as a polygon or a polyhedron), we decide if a direction of formation exists that will result in the successful construction of the object. Such a direction will be called a valid direction of formation. We provide an $O(n)$ time algorithm for finding a valid direction of formation where $n$ is the number of vertices of the object. Furthermore, if the object is feasible, we report a description of all the orientations in which the object can be made. We then define a more flexible model that more accurately reflects the actual capabilities of stereolithography (referred to as variable-angle stereolithography). In this model, we assume that as each layer is welded on to the previous, the top layer may hang over the previous by a certain fixed amount. Again we study the class of feasible objects for this model. We give an $O(n)$ time algorithm for polygons and $O(n \log n)$ as well as $O(n)$ time algorithms for polyhedra.

We show that objects formed using variable-angle stereolithography can also be constructed using gravity casting ([5], [6], [7], [13]). We also show that the polyhedral objects formed by vertical stereolithography are closely related to polyhedral terrains which are important structures in geographic information systems ([15], [16]) and computational geometry ([8], [11]). In fact, our algorithms recognize whether a polyhedral surface is a terrain that allows overhangs, thus initiating the study of more realistic terrains than the standard ones considered in geographic information systems. Finally, we show that the polygonal and polyhedral objects built by vertical stereolithography can be clamped by parallel jaw grippers with any positive sized gripper.

\section{Notation and Preliminaries}

The objects studied are simple polygons and simple polyhedra as defined in [22]. The object will always be oriented so that the direction of formation being discussed is the negative $y$-direction. 
Therefore a direction of formation defines the orientation of the object.

A given direction $\Theta$ in the plane will be specified by a point on a unit circle in the following way. Let $C$ be a unit circle with center $o$. Any point $x$ on the boundary of the circle represents the direction $\overrightarrow{o x}$ (Refer to Figure 3 ). The point $x$ will either be considered as the unit vector $\overrightarrow{o x}$ or the point on the unit circle depending on the context of its usage. A point that is diametrically opposite to $x$ on the unit circle represents the inverse or opposite direction to direction $x$ and is denoted by $\operatorname{opp}(x)$. A right normal to direction $x$ is denoted by $\mathrm{N}^{+}(x)$ and a left normal by $\mathrm{N}^{-}(x)$.

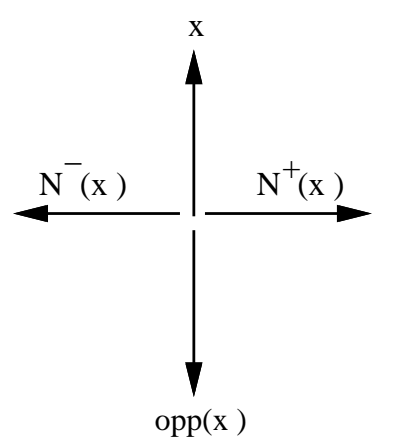

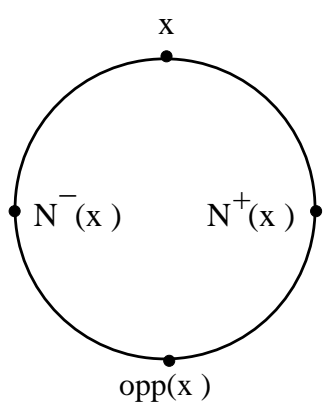

Directions on the unit circle

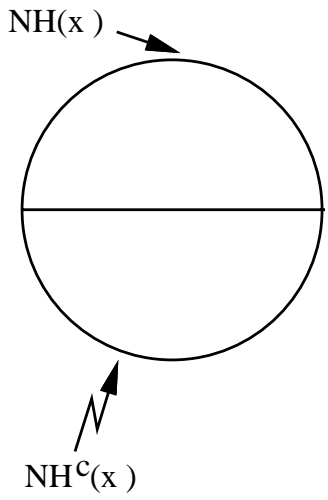

Figure 3: Mlustrating the representation of directions.

An equivalence class of parallel lines $H$ in the plane will be specified by a pair of points $p_{1}$ and $p_{2}$ that are diametrically opposite on the unit circle, such that the line determined by the two points is parallel to a line in $H$. A normal to a line in $H$ is an equivalence class $N$ of parallel lines with the property that every member of $N$ is orthogonal to the lines in $H$. Given a direction $x$, we define the normal to $x$, denoted by $N(x)$, as the equivalence class of parallel lines that are orthogonal to $x$. Notice that the two points representing the normal divide the boundary of the unit circle into two semi-circles. We refer to the open semi-circle containing the point representing the direction $x$ as the open normal semi-circle or the open normal half-plane of $x$ and denote it as $N H(x)$. The closed semi-circle is denoted by $N H[x]$. The open semi-circle not containing the point representing the direction $x$ will be denoted by $N H^{c}(x)$. Similarly, the closed semi-circle not containing the point is $N H^{c}[x]$.

Given two points $a$ and $b$ in the plane, let $[a b]$ and $(a b)$ denote, respectively, the closed and open line segments between the two points. Given a line segment $e$ in the plane, we denote the line containing $e$ by $L(e)$. Given two points $a$ and $b$ on the unit circle, let $\operatorname{arc}[a, b]$ and $\operatorname{arc}(a, b)$ denote respectively the closed and open arcs of the unit circle from $a$ to $b$ in the clockwise direction.

Similarly, we represent the set of all directions in 3 -space by the points on the surface of a unit sphere (see Figure 4 for definitions to follow). Let $\mathcal{S}$ be the unit sphere centered at the origin $o$. Any point $p$ on $\mathcal{S}$ represents the direction $\overrightarrow{o p}$. A point that is diametrically opposite to $p$ on the unit sphere represents the inverse or opposite direction to direction $p$ and is denoted by opp $(p)$. Notice that all the points $s$ on the boundary of $\mathcal{S}$ with the property that $\overrightarrow{o s} \cdot \overrightarrow{o p}=0$ (- represents the inner product) form a great circle. We denote this great circle by $N(p)$ since all these points 
are directions that are orthogonal to $p$. The great circle $N(p)$ divides the sphere into two halfspheres. The open half-sphere containing $p$ will be denoted as $N H(p)$ and the closed half-sphere by $N H[p]$. The open and closed half-spheres not containing $p$ will be denoted by $N H^{c}(p)$ and $N H^{c}[p]$, respectively. When considering the angle between two vectors, we always mean the smaller angle unless stated otherwise.

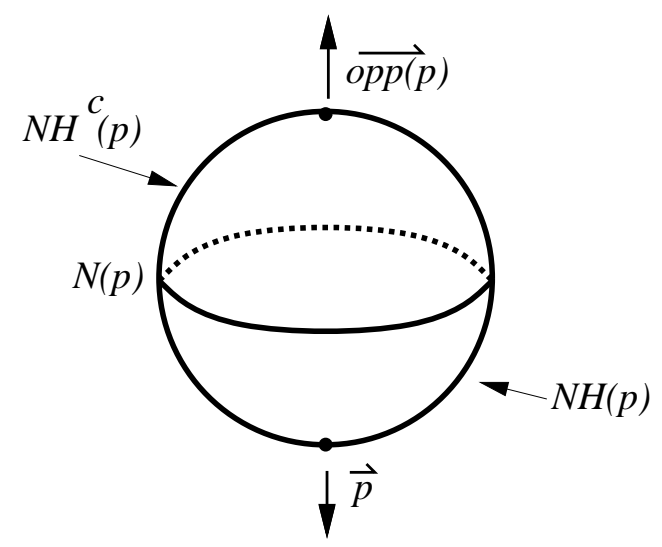

Figure 4: The sphere of directions.

\section{$3 \quad$ Vertical Stereolithography}

We first define the geometric model of stereolithography referred to as vertical stereolithography.

A polygonal object is assumed to rest on the $x$-axis and a polyhedral object is assumed to lie on the plane defined by $y=0$. For a given object $A$ and direction of formation $d$, let $A_{d}$ denote the object oriented and positioned according to $d$. For $y_{0} \geq 0$, let $A_{d}\left(y_{0}\right)$ be the intersection of $A_{d}$ with the line $y=y_{0}$ for polygonal objects and the plane $y=y_{0}$ for polyhedral objects. We refer to $A_{d}(0)$ as the base of the object (with respect to $d$ ). A point $p$ of the object with $y$-coordinate $y_{0}$ is said to be supported (with respect to a particular direction of formation) if all the points with $x$ (and $z$ ) coordinates the same as $p$ and positive $y$ coordinate less than $y_{0}$ are in the object. The cross-sections of the object are assumed to be infinitesimally thin and so direction $d$ is a valid direction of formation for an object if the resulting orientation of the object is such that all points in the object are supported. An object is referred to as feasible provided it has at least one valid direction of formation.

\subsection{Polygonal Objects}

In this subsection we consider the two-dimensional problem where the object $A$ we wish to form under the vertical stereolithography model is a simple polygon. Let $v_{0}, v_{1}, \ldots, v_{n-1}$ be the clockwise ordering of the vertices around $A$ such that each pair of consecutive vertices $v_{i}, v_{i+i}$ is joined by an edge $\boldsymbol{e}_{i}$ (all indicies are taken modulo $n$ ). For $1 \leq i \leq n$, let $\theta_{i}$ be the angle formed by $\boldsymbol{e}_{i-1}$ and $\boldsymbol{e}_{i}$ in the interior of $A$. If edge $e_{i}$ is such that $\theta_{i+1}$ and $\theta_{i}$ are both less than or equal to $\pi / 2$ then $e_{i}$ 
is called an acute edge. If $e_{i}$ is an acute edge and at least one of $\theta_{i+1}$ or $\theta_{i}$ is strictly less than $\pi / 2$ then $e_{i}$ is said to be a strictly acute edge. Let $n_{i}$ denote the direction normal to edge $e_{i}$ pointing out of the polygon. Let $\mathcal{N}$ be the set of all outer normals. We will denote the open interior of the polygon $A$ by $\operatorname{int}(A)$, the boundary by $b d(A)$, and the open exterior by $\operatorname{ext}(A)$. The boundary is considered part of the polygon; that is, $A=\operatorname{int}(A) \cup b d(A)$.

We first observe a simple geometric fact that will be useful in establishing many of the lemmas and theorems to follow. Let $e_{i}$ be an edge of polygon $A$. Let $p$ be a point on the open edge $e_{i}$. Let $r$ be a ray emanating from point $p$ in direction $d$.

Observation 3.1 There exists a point $q \in r$ distinct from $p$ such that $(p q)$ is contained in ext $(A)$ if and only if $d \cdot n_{i}$ is positive (i.e. the angle between $d$ and $n_{i}$ is strictly less than $\pi / 2$ ).

We begin by showing that the base of a feasible object must be an edge.

Lemma 3.1 If $d$ is a valid direction of formation for polygon $A$, then $A_{d}(0)$ is some edge of $A$.

Proof: If $A_{d}(0)$ is not an edge, then it must be a vertex, say $v_{i}$. Since both $v_{i-1}$ and $v_{i+1}$ are above the line $y=0$, at least one of the two cannot be supported by Observation 3.1 .

The above lemma restricts our search for a valid direction of formation to the outer normals of the edges of a polygon, namely the set $\mathcal{N}$. Therefore, edge $e_{i}$ of polygon $A$ is said to be a valid base if $n_{i}$ is a valid direction of formation. A point $p$ in $A_{n_{i}}$ is said to be vertically visible from $e_{i}$ if the vertical line segment from $p$ to $\epsilon_{i}$ is contained in $A_{n_{i}}$. Thus, we observe the following

Observation 3.2 A polygon $A_{n_{i}}$ is feasible with valid base $e_{i}$ if and only if all points in $A_{n_{i}}$ are vertically visible from $e_{i}$.

Although Observation 3.2 provides some insight into the structure of a feasible polygon, the following characterization of feasible polygons is useful from a computational perspective.

Lemma 3.2 An edge $e_{i}$ of $A_{n_{i}}$ is a valid base if and only if $n_{i} \cdot n_{j} \leq 0(\forall 1 \leq j \leq n, j \neq i)$.

\section{Proof:}

$(\Rightarrow)$ Suppose $e_{i}$ is a valid base but there exists an edge $e_{j}$ such that $n_{i} \cdot n_{j}>0$. Consider a point $p$ on the open edge $e_{j}$. Let $q$ be the orthogonal projection of $p$ onto the line $L\left(e_{i}\right)$. The open line segment $(p q)$ must be contained in $A_{n_{i}}$. However, this is impossible by Observation 3.1.

$(\Leftarrow)$ Suppose that $n_{i} \cdot n_{j} \leq 0(\forall 1 \leq j \leq n, j \neq i)$, but $e_{i}$ is not a valid base. Then there must exist some point $p$ in $A_{n_{i}}$ that is not vertically visible from $e_{i}$ by Observation 3.2. Let $q$ be the orthogonal projection of $p$ onto $L\left(e_{i}\right)$. Line segment $[p q]$ must intersect $b d\left(A_{n_{i}}\right)$ above $L\left(e_{i}\right)$ since $p$ is not vertically visible from $e_{i}$. Let $x$ be the intersection point of $[p q]$ and $b d\left(A_{n_{i}}\right)$ closest to $p$. Let us assume for the moment that $x$ is on the open edge $e_{k}$. Line segment $[p x]$ must be in $A_{n_{i}}$ since $p$ is in $A_{n_{i}}$ and $x$ is the first intersection with the boundary. Let $y$ be the intersection of $[x q]$ with $b d\left(A_{n_{i}}\right)$ closest to $x$ or $q$ if no such intersection exists. Line segment $(x y)$ is contained in $\operatorname{ext}\left(A_{n_{i}}\right)$. But this implies that $n_{k} \cdot n_{i}>0$ by Observation 3.1 which is a contradiction. A similar argument holds had $x$ been a vertex. 
With this in mind, we uncover a key characteristic of valid bases, that leads to a linear time algorithm.

Lemma 3.3 If $e_{i}$ is a valid base then $e_{i}$ is acute.

Proof: Suppose $e_{i}$ is a valid base that is not acute. Then either $n_{i-1} \cdot n_{i}>0$ or $n_{i+1} \cdot n_{i}>0$ or both. By Lemma 3.2 this contradicts the fact that $e_{i}$ is valid.

Given this characteristic, we completely characterize the convex objects that are feasible. The following lemma shows that for a convex object $A$ there is a simple linear time test to find a valid base for $A$ or report that none exists.

Lemma 3.4 Given a convex polygon $A$, the edge $e_{i}$ is a valid base if and only if $e_{i}$ is acute.

\section{Proof:}

$(\Rightarrow)$ If $\epsilon_{i}$ is a valid base, then by Lemma 3.3 it must be acute.

$(\Leftarrow)$ Since $e_{i}$ is acute, extending $\epsilon_{i-1}$ and $\epsilon_{i+1}$ causes them to meet at a point directly above some point of $e_{i}$, thus forming a triangle with $e_{i}$ that is vertically visible from $e_{i}$. By convexity, $A$ must lie in this triangle and so for any point $p$ in $A$ there is a point $q$ on $e_{i}$ vertically below $p$. Therefore, by Observation $3.2, e_{i}$ is a valid base.

The characterization of convex objects in Lemma 3.4 implies that a simple examination of the angles between the edges of a convex object is sufficient to find a valid base if one exists or report that the object is not feasible. For a non-convex object, such local tests on the angles are insufficient to determine the feasibility of an object, since such an object may have an acute edge that is not a valid base. For example, in Figure 5, edge $e_{i}$ is an acute edge but not a valid base of the polygon since vertex $v_{i-2}$ is not supported. However, the following lemma shows the relationship between the feasibility of a simple polygon and its convex hull.

Lemma 3.5 If simple polygon $A_{n_{i}}$ is feasible with base $e_{i}$ then the convex hull of $A_{n_{i}}$ is also feasible with base $e_{i}$.

Proof: Follows from Observation 3.2, Lemma 3.3 and Lemma 3.4.

Since the convex hull of a simple polygon can be computed in linear time [17, 18], coupled with the fact that a convex polygon can only have at most 4 acute edges, we see that feasibility of a simple polygon can be computed in linear time. The convex hull of a simple polyhedron, however, cannot be computed in linear time, but can be computed in $O(n \log n)$ time (see [22]). Therefore, although this approach provides an optimal solution to the problem in two dimensions, a solution in three dimensions will require an additional $\log n$ factor. To this end, we explore the following alternate solution that can be generalized to the three-dimensional version of the problem.

Let us first examine the restrictions that the existence of a strictly acute edge puts on the feasibility of a non-convex polygon. 


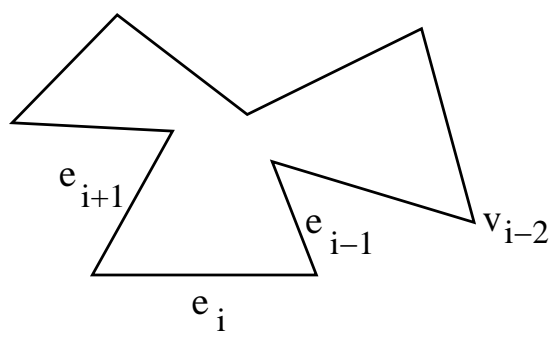

Figure 5: A non-convex object with an acute edge that is not a valid base.

Lemma 3.6 If a simple polygon $A$ is feasible and edge $e_{i}$ of $A$ is strictly acute then the set of all valid bases of $A$ is a non-empty subset of $\left\{e_{i}, e_{i-1}, e_{i+1}\right\}$.

Proof: (Refer to Figure 5). Suppose that none of $e_{i}, e_{i-1}$ and $e_{i+1}$ are valid. Since $e_{i}$ is strictly acute, without loss of generality, assume that $\theta_{i}<\pi / 2$. Since $A$ is feasible, let $e_{j}$ be a valid base of $A$. Notice that $n_{j}$ cannot be contained in $N H\left(n_{i}\right)$ since otherwise $n_{j} \cdot n_{i}>0$. Similarly, $n_{j}$ cannot be in $\operatorname{arc}\left[N^{+}\left(n_{i}\right), \operatorname{opp}\left(n_{i}\right)\right)$ because otherwise $n_{j} \cdot n_{i+1}>0$. Also, $n_{j}$ cannot be in $\operatorname{arc}\left[\operatorname{opp}\left(n_{i}\right), N^{-}\left(n_{i}\right)\right]$ since otherwise $n_{j} \cdot n_{i-1}>0$. But $N H\left(n_{i}\right) \cup \operatorname{arc}\left[N^{+}\left(n_{i}\right), \operatorname{opp}\left(n_{i}\right)\right) \cup \operatorname{arc}\left[\operatorname{opp}\left(n_{i}\right), N^{-}\left(n_{i}\right)\right]$ represents all directions. Therefore, $n_{j}$ cannot exist.

Lemma 3.3 guarantees that an acute edge $e_{i}$ exists if $A$ is feasible and Lemma 3.6 says that if a strictly acute edge $e_{i}$ exists then it is sufficient to test $e_{i}, e_{i-1}$ and $e_{i+1}$ for a valid base. We now consider what happens when $\epsilon_{i}$ is an acute edge with both $\theta_{i+1}$ and $\theta_{i}$ are equal to $\pi / 2$. If $A_{n_{i}}$ contains a single edge $\epsilon_{j}$ such that $n_{j}$ is $\operatorname{opp}\left(n_{i}\right)$ then we label the edge $\boldsymbol{e}_{\text {top }}(i)$.

Lemma 3.7 If $A$ is feasible and $e_{i}$ is an acute edge such that $\theta_{i+1}=\theta_{i}=\pi / 2$ then the set of all valid bases of $A$ is a non-empty subset of $\left\{e_{i}, e_{i-1}, e_{i+1}, e_{t o p}(i)\right.$ (if it exists) $\}$.

Proof: Similar to the proof of Lemma 3.6.

With Lemma 3.7 we have characterized all polygons that are feasible. We summarize with the following theorem.

Theorem 3.1 Given that $A$ contains an acute edge $e_{i}$, the set of all valid bases of $A$ is a nonempty subset of $\left\{e_{i}, e_{i-1}, e_{i+1}, e_{t o p}(i)\right.$ (if it exists and $\left.\left.\theta_{i+1}=\theta_{i}=\pi / 2\right)\right\}$ if and only if polygon $A$ is feasible.

Proof: Follows from Lemma 3.3, Lemma 3.6, and Lemma 3.7.

Determining whether or not a polygon has an acute edge can be achieved in $O(n)$ time, where $n$ is the number of vertices of the polygon. Thus, in $O(n)$ time, the number of possible valid bases can be reduced to 3 or 4 by Theorem 3.1. Moreover, by Lemma 3.2 we can test in $O(n)$ time whether 
any of these candidate edges is valid simply by testing its outward normal with the outward normals of all the other edges. Therefore, we can test a polygon $A$ for feasibility and find all valid bases in $O(n)$ time.

Theorem 3.2 In $O(n)$ time the feasibility of a polygonal object with $n$ vertices can be determined and all valid bases identified when the object is feasible.

\subsection{Polyhedral Objects}

In this subsection we consider the three-dimensional case where the object is a simple polyhedron. We want to find a face of polyhedron $A$ that is a valid base or determine that $A$ is not feasible.

The following notation will be used in this subsection. Let $A$ be a polyhedron with $n$ vertices. Given a face $f$ of a polyhedron, we denote the plane containing $f$ by $P(f)$. For face $f$ of $A$, let $f(1), f(2), \ldots, f\left(k_{f}\right)$ be the faces of $A$ that share at least one edge with $f$. Let $\theta_{i}(f)$ be the angle interior to $A$ between the plane $P(f)$ and the plane $P(f(i))$ about the line of intersection of $P(f)$ and $P(f(i))$. If $\theta_{i}(f) \leq \pi / 2$ for all $i, 1 \leq i \leq k_{f}$, then $f$ is called an acute face. If $f$ is acute and for some $i, \theta_{i}(f)<\pi / 2$, then $f$ is said to be a strictly acute face. Let $n(f)$ denote the direction normal to face $f$ pointing out of the polyhedron. Let $\mathcal{N}$ be the set of all outer normals. We will denote the open interior of the polyhedron $A$ by int $(A)$, the boundary by $b d(A)$, and the open exterior by $\operatorname{ext}(A)$. The boundary is considered part of the polyhedron; that is, $A=\operatorname{int}(A) \cup b d(A)$. We show several properties analogous to those in the previous subsection that will give rise to a linear time feasibility testing algorithm. We first observe a simple geometric fact. Let $f$ be a face of polyhedron $A$. Let $p$ be a point on the open face $f$. Let $r$ be a ray emanating from point $p$ in direction $d$.

Observation 3.3 There exists a point $q \in r$ distinct from $p$ such that $(p q)$ is contained in ext $(A)$ if and only if $d \cdot n(f)$ is positive (i.e. the angle between $d$ and $n(f)$ is strictly less than $\pi / 2$ ).

We begin by showing that the base of a feasible object must be a face.

Lemma 3.8 If $d$ is a valid direction of formation for polyhedron $A$, then $A_{d}(0)$ is some face of $A$.

Proof: If $A_{d}(0)$ is not a face, then it must either be an edge or a vertex. If it is an edge $e$, then let $f_{i}$ and $f_{j}$ be the two faces adjacent to $e$. Since both faces lie above a plane containing $e$, either $n\left(f_{i}\right) \cdot d$ or $n\left(f_{j}\right) \cdot d$ is positive. Without loss of generality, assume it to be $n\left(f_{i}\right)$. By Observation 3.3 there is a point on the open face $f_{i}$ that is not supported. A similar argument holds if $A_{d}(0)$ is a vertex.

The above lemma restricts our search for a valid direction of formation to the outer normals of the faces of a polyhedron, namely the set $\mathcal{N}$. Therefore, face $f$ of polyhedron $A$ is said to be a valid base provided that $n(f)$ is a valid direction of formation. A point $p$ in $A_{n(f)}$ is said to be vertically visible from $f$ if the vertical line segment from $p$ to $f$ is contained in $A_{n(f)}$. Thus, we observe the following.

Observation 3.4 A polyhedron $A_{n(f)}$ is feasible with valid base $f$ if and only if all points in $A_{n(f)}$ are vertically visible from $f$. 
As in the two dimensional case, the following characterization of feasible polyhedra will prove to be more useful from a computational perspective.

Lemma 3.9 A face $f_{i}$ of $A_{n\left(f_{i}\right)}$ is a valid base if and only if $n\left(f_{i}\right) \cdot n\left(f_{j}\right) \leq 0$ (for all faces $f_{j}$ of $A_{n\left(f_{i}\right)}$, where $\left.f_{j} \neq f_{i}\right)$.

\section{Proof:}

$(\Rightarrow)$ Suppose $f_{i}$ is a valid base but there exists a face $f_{j}$ such that $n\left(f_{i}\right) \cdot n\left(f_{j}\right)>0$. Consider a point $p$ on the open face $f_{j}$. Let $q$ be the orthogonal projection of $p$ onto the plane $P\left(f_{i}\right)$. The line segment $[p q]$ must be contained in $A_{n\left(f_{i}\right)}$. However, this is impossible by Observation 3.3.

$(\Leftarrow)$ Suppose that $n\left(f_{i}\right) \cdot n\left(f_{j}\right) \leq 0$ for all faces $f_{j}$ of $A_{n\left(f_{i}\right)}$ distinct from $f_{i}$, but $f_{i}$ is not a valid base. Then there must exist some point $p$ in $A_{n\left(f_{i}\right)}$ that is not vertically visible from $f_{i}$ by Observation 3.4. Let $q$ be the orthogonal projection of $p$ onto $P\left(f_{i}\right)$. Line segment $[p q]$ must intersect $b d\left(A_{n\left(f_{i}\right)}\right)$ above $P\left(f_{i}\right)$ since $p$ is not vertically visible from $f_{i}$. Let $x$ be the intersection point of $[p q]$ and $b d\left(A_{n\left(f_{i}\right)}\right)$ closest to $p$. Let us assume for the moment that $x$ is on the open face $f_{j}$. Line segment $[p x]$ must be in $A_{n\left(f_{i}\right)}$ since $p$ is in $A_{n\left(f_{i}\right)}$ and $x$ is the first intersection with the boundary. Let $y$ be the intersection of $[x q]$ with $b d\left(A_{n\left(f_{i}\right)}\right)$ closest to $x$ or $q$ if no such intersection exists. Line segment $(x y)$ is contained in $\operatorname{ext}\left(A_{n\left(f_{i}\right)}\right)$. But this implies that $n\left(f_{j}\right) \cdot n\left(f_{i}\right)>0$ by Observation 3.3 which is a contradiction. A similar argument holds for the case where $x$ is a vertex or on an edge.

Lemma 3.10 If face $f$ is a valid base for polyhedron $A$ then $f$ is acute.

Proof: Suppose that $f$ is a valid base for $A$ but $f$ is not acute. Then there must be some $f(i)$ such that $\theta_{i}(f)>\pi / 2$. However, this implies that $n(f) \cdot n(f(i))>0$. By Lemma 3.9, this contradicts the fact that $f$ is valid.

In the special case of convex polyhedra, we see that a simple local test on each face suffices to determine if a face is a valid base.

Lemma 3.11 Let $A$ be a convex polyhedron. Face $f$ is a valid base if and only if $f$ is acute.

Proof: Similar to proof of Lemma 3.4.

It is no longer clear whether the feasibility of a convex polyhedron can be determined in $O(n)$ time since a face $f$ of a polyhedron may have $O(n)$ adjacent faces. However, the total complexity of all adjacencies is linear by Euler's formula (see [3]). Therefore, testing all faces for validity by the local test implied in Lemma 3.11 can be done in $O(n)$ time. We now turn our attention to polyhedral objects that are not necessarily convex. The following lemma shows the relationship between the feasibility of a simple polyhedron and its convex hull.

Lemma 3.12 If simple polyhedron $A_{n(f)}$ is feasible with base $f$ then the convex hull of $A_{n(f)}$ is also feasible with base $f$. 


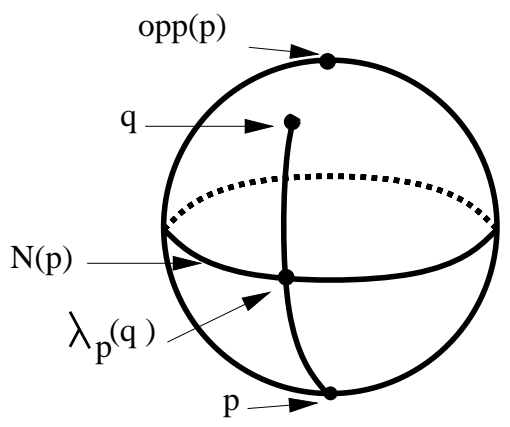

Figure 6: Illustrating $\lambda_{p}(q)$

Proof: Follows from Observation 3.4, Lemma 3.10, and Lemma 3.11.

Lemma 3.12 implies the following simple approach to determine if a given polyhedron $A$ is feasible. Compute the convex hull of $A$ in $O(n \log n)$ time. A convex polyhedron can have at most 6 acute faces. Each acute face of the convex hull is a candidate base. Testing a face can be done in linear time by Lemma 3.9. Therefore, determining feasibility of a simple polyhedron can be achieved in $O(n \log n)$ time. The complexity is dominated by the computation of the convex hull. To circumvent the computation of the convex hull, we explore the following approach which will lead to an optimal algorithm.

We first examine the restrictions placed on the feasibility of a polyhedron in the presence of a strictly acute face. Before doing so, we define the following geometric term (see Figure 6). Let $p$ be a point on the sphere of directions $\mathcal{S}$. Let $q$ be any point on $\mathcal{S}$ distinct from $p$ and $o p p(p)$. We define $\lambda_{p}(q)$ to be the point on $N(p)$ closest to $q$ (i.e. the intersection point closest to $q$ of $N(p)$ with the great circle through $p$ and $q$ ).

We show that if the polyhedral object $A$ has a strictly acute face $f$, then $f$ or one of its adjacent faces must be a valid base if the object is feasible.

Lemma 3.13 If polyhedron $A$ is feasible and $f$ is a strictly acute face then the set of all valid bases of $A$ is a non-empty subset of $\left\{f, f(1), \ldots, f\left(k_{f}\right)\right\}$.

Proof: Suppose that none of $f, f(1), \ldots$ and $f\left(k_{f}\right)$ are valid. Since $f$ is strictly acute, without loss of generality, assume that $\theta_{i}(f)<\pi / 2$. Since $A$ is feasible, let $f_{j}$ be a valid base of $A$. We see that $n\left(f_{j}\right) \neq \operatorname{opp}(n(f))$ since $n(f(i)) \cdot o p p(n(f))>0$. This implies that $\lambda_{n(f)}\left(n\left(f_{j}\right)\right)$ is properly defined. Now, we know that $n\left(f_{j}\right)$ cannot be in $N H(n(f))$ for this would violate the validity of face $f_{j}$ by Lemma 3.9. Therefore, $n\left(f_{j}\right)$ must be in $N H^{c}[n(f)]$.

We notice that $\lambda_{n(f)}(n(f(i))$ is simply the outward normal of the edge of face $f$ (which is a polygon) corresponding to the intersection of $f(i)$ and $f$. It follows that every open half-circle $\subset N(n(f))$ contains at least one point of $\lambda_{n(f)}(f(1)), \lambda_{n(f)}(f(2)), \ldots$, or $\lambda_{n(f)}\left(f\left(k_{f}\right)\right)$ since face $f$ is a simple polygon. Therefore, given a point $x \neq o p p(n(f))$ in $N H^{c}[n(f)]$, there exists a face $f(i)$ adjacent to $f$ such that $\lambda_{n(f)}(x) \cdot \lambda_{n(f)}\left(n\left(f_{i}\right)\right)>0$. Observe, however, that if two directions 
$a, b \in N H^{c}[n(f)]$ both distinct from $\operatorname{opp}(n(f))$ are such that $\lambda_{n(f)}(a) \cdot \lambda_{n(f)}(b)>0$, then $a \cdot b>0$. But this implies that $n\left(f_{j}\right)$ cannot exist.

If $f$ is not strictly acute, we define $f_{\text {top }}$ analogously to $e_{\text {top }}(i)$ in the previous subsection. We have the following lemma.

Lemma 3.14 If polyhedron $A$ is feasible and $f$ is an acute, but not strictly acute, face then the set of all valid bases of $A$ is a non-empty subset of $\left\{f, f(1), \ldots, f\left(k_{f}\right), f_{\text {top }}\right.$ (if it exists) $\}$.

Proof: Similar to the argument given in the proof of Lemma 3.13.

These results were sufficient in the two-dimensional case to reduce the number of candidate bases to at most 4. Unfortunately, in the 3-dimensional case, an acute face $f$ may have $O(n)$ adjacent faces. However, we are able to link the feasibility of a face in a polyhedron to the feasibility of an edge in a polygon. Thus, we establish the following theorem.

Theorem 3.3 Given that $A$ has an acute face $f$, polyhedron $A$ is feasible if and only if the set of all valid bases of $A$ is a non-empty subset of $\left\{f, f_{\text {top }}\right.$ (if it exists) and at most 4 faces adjacent to $f\}$. Moreover, the edges corresponding to the intersection of $f$ with the at most 4 faces adjacent to $f$ are valid edges for polygon $f$.

\section{Proof:}

$(\Rightarrow)$ If the set of valid bases of $A$ is a non-empty subset of: $f, f_{\text {top }}$ (if it exists) and at most 4 faces adjacent to $f$, then by definition, $A$ is feasible.

$(\Leftarrow)$ If $A$ is feasible, we must show that the following faces of $A$ are the only valid bases: $f$, $f_{\text {top }}$ (if it exists) and at most 4 faces adjacent to $f$. Lemma 3.14 reduces our task to showing that at most 4 faces adjacent to $f$ can be bases. Suppose 5 faces adjacent to $f$ were valid bases. Let us denote them by $f\left(i_{1}\right), f\left(i_{2}\right), \ldots, f\left(i_{5}\right)$. Notice that $n\left(f\left(i_{1}\right)\right), n\left(f\left(i_{2}\right)\right), n\left(f\left(i_{3}\right)\right), n\left(f\left(i_{4}\right)\right)$, and $n\left(f\left(i_{5}\right)\right)$ are all contained in $N H^{c}[n(f)]$ since $f$ is acute. Also, since they are all valid bases, $n\left(f\left(i_{j}\right)\right) \cdot n\left(f_{k}\right) \leq 0$ for all $1 \leq j \leq 5$ and for all faces $f_{k} \neq f\left(i_{j}\right)$ of $A$ by Lemma 3.9.

Let $f(1), f(2), \ldots, f\left(k_{f}\right)$ be the faces adjacent to face $f$. Since $f$ is acute $n(f(1)), \ldots, n\left(f\left(k_{f}\right)\right)$ are all contained in $N H^{c}[n(f)]$. Observe that $\lambda_{n(f)}\left(n(f(k))\right.$ is properly defined for all $1 \leq k \leq k_{f}$. Since each of $f\left(i_{1}\right), f\left(i_{2}\right), \ldots, f\left(i_{5}\right)$ is a valid base, we have that $\lambda_{n(f)}\left(n\left(f\left(i_{j}\right)\right)\right) \cdot \lambda_{n(f)}(n(f(k)) \leq 0$ for all $1 \leq j \leq 5$ and all faces $f(k)$ adjacent to $f$ distinct from $f\left(i_{j}\right)$. We notice that $\lambda_{n(f)}(n(f(k))$ is simply the outward normal of the edge of face $f$ (which is a polygon) corresponding to the intersection of $f(k)$ and $f$. But this would mean that polygon $f$ has 5 valid edges by Lemma 3.2 , contradicting Theorem 3.1 .

Therefore, the number of possible valid bases in a feasible polyhedron $A$ is at most 6 . We summarize below the linear time algorithm to determine the feasibility of a simple polyhedron. The algorithm takes a simple polyhedron $A$ as input. 
Algorithm: Determine the feasibility of a simple polyhedron.

1. Determine if $A$ has an acute face. If $A$ does not have an acute face, exit ( $A$ is not feasible).

2. Let $f$ be the acute face of $A$. Scan all other faces of $A$ to determine if $f_{\text {top }}$ exists.

3. Compute all possible valid edges of polygon $f$ using the algorithm described in Section 3.1. There are at most 4 edges. Let $F^{\prime}$ represent the faces of $A$ adjacent to these edges excluding face $f$.

4. Let $B$ be $\left\{f, f_{\text {top }}\right.$ (if it exists) $\} \cup F^{\prime}$. The set $B$ represents the candidate bases of $A$. There are at most 6 faces in $B$ by Theorem 3.3.

5. Test each face $f_{i} \in B$ to see if it is valid in the following way:

Check that the angle between normal $n\left(f_{i}\right)$ and all other normals is no less than $\pi / 2$. This can be done in linear time.

6. Output the valid bases.

The correctness of the algorithm follows from Theorem 3.3. As for the time complexity, we see that step 1 can be done in $O(n)$ time by Euler's formula (see [3]). Step 3 takes linear time by the algorithm given in Section 3.1. Furthermore, by Lemma 3.9 testing each candidate face can be done in $O(n)$ time simply by testing its outward normal with the outward normals of all the other faces. Since there are only a maximum of 6 candidate faces, we conclude that testing a polyhedron $A$ for feasibility and finding all valid bases can be achieved in $O(n)$ time.

Theorem 3.4 In $O(n)$ time the feasibility of a polyhedral object with $n$ vertices can be determined and all valid bases identified when the object is feasible.

\section{Variable-Angle Stereolithography}

In practice, as the laser welds one cross-section on to the other, if the top layer is "close enough" to the previous layer, it can be welded on. That is, the upper layer may hang over the previous by a certain amount and still get welded on. To model this mathematically, we define the following model referred to as variable-angle stereolithography.

Intuitively, variable-angle stereolithography differs from vertical stereolithography in the following way. As each layer is glued on by the laser, the topmost layer can hang over the previous layer by the freedom allotted by some constant angle $\omega$. More formally, we say that a point $p$ with $y$-coordinate $y_{0}$ is $\omega$-supported with respect to the direction of formation if there exists a point $q$ with positive $y$ coordinate less than $y_{0}$ such that the line segment $[p q]$ is contained in the object and the smaller angle between the direction of formation and the vector $\overrightarrow{p q}$ is less than or equal to $\omega$. Clearly, $\omega$ must be less than $\pi / 2$. Notice that variable-angle stereolithography is a generalization of vertical stereolithography. The two are equivalent when $\omega$ is zero. An object can be built with respect to the parameter $\omega$ if there exists an orientation of the object such that all points above the base are $\omega$-supported. An object that can be built with respect to the parameter $\omega$ will be called $\omega$-feasible. 


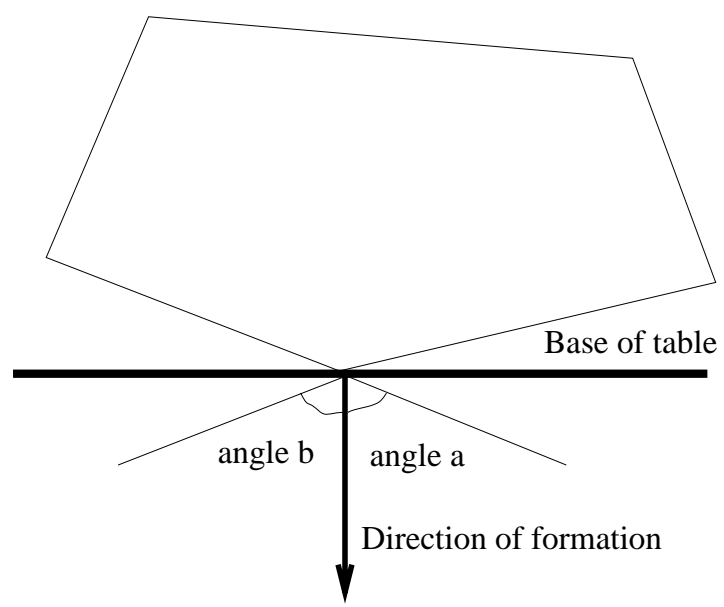

Figure 7: A vertex that is a valid base.

\subsection{Polygonal Objects}

The parameter $\omega$ enlarges the class of objects that can be formed. In fact, with $\omega>0$, the base of an object no longer need be a edge of the polygon. For example, the polygon in Figure 7 is feasible (as long as both $\angle a$ and $\angle b$ are both less than or equal to $\omega$ ) with a vertex as base. For polygonal objects, we will assume that the base of an object is always an edge, since building an object on a vertex is unstable.

We say that a point $p$ in $A_{n_{i}}$ is $\omega$-visible from $e_{i}$ if $p$ is above $L\left(e_{i}\right)$ and there exists a polygonal path $\Pi$ from $p$ to $e_{i}$ such that $\Pi \in A_{n_{i}}$ and every vertex in $\Pi$ (except for the vertex on $e_{i}$ ) is $\omega$-supported by an adjacent vertex. Thus, we observe the following.

Observation 4.1 A polygon $A_{n_{i}}$ is $\omega$-feasible with valid base $e_{i}$ if and only if all points in $A_{n_{i}}$ are $\omega$-visible from $e_{i}$.

A polygonal chain is said to be monotonic with respect to direction $\Theta$ if the intersection of every line parallel to $N(\Theta)$ with the chain is either empty or a point. We observe the following property that is crucial to the development of a linear algorithm.

Observation 4.2 If a point $p$ is $\omega$-visible from $e_{i}$, then there exists a path $\Pi$ from $p$ to $e_{i}$ that is monotone with respect to direction $n_{i}$.

We present an alternate characterization of $\omega$-feasibility that will be useful from a computational perspective.

Theorem 4.1 A polygon $A_{n_{i}}$ is $\omega$-feasible with valid base $e_{i}$ if and only if the angle between $n_{i}$ and all other normals is no less than $\pi / 2-\omega$ and the set of all local minima with respect to $n_{i}$ is $e_{i}$. 


\section{Proof:}

$(\Leftarrow)$ Given that all $n_{j}$ distinct from $n_{i}$ are such that the smaller angle between $\angle n_{i} n_{j} \geq \pi / 2-\omega$, and all local minima are contained in $e_{i}$, we will show that $A_{n_{i}}$ is $\omega$-feasible with base $e_{i}$. We do this by showing that every point in $A_{n_{i}}$ is $\omega$-supported by the following construction.

Let $p \in A_{n_{i}}$. Assume that $p \notin e_{i}$.

1. If $p$ is contained in $\operatorname{int}\left(A_{n_{i}}\right)$, then let $q$ be the intersection point below $p$ and closest to $p$ of a vertical line through $p$ and $b d\left(A_{n_{i}}\right)$.

2. If $p$ is contained in the interior of an edge $e$, then let $q$ be the vertex adjacent to $e$ with lower $y$-coordinate. Such a vertex must exist since $p$ is not a local minimum.

3. If $p$ is a vertex $v$, then let $q$ be the vertex adjacent to $v$ with lower $y$-coordinate. Such a vertex must exist since $p$ is not a local minimum.

By construction, the smaller angle between $\overrightarrow{p q}$ and $n_{i}$ is no more than $\omega$. Therefore, $p$ is $\omega$ supported. If $q \in e_{i}$, then we are done. If $q \notin e_{i}$, we must show that $q$ is $\omega$-supported. This can be done by repeating steps $1,2,3$ with $q$. The construction must end with a point on $e_{i}$ since $e_{i}$ contains all local minima with respect to $n_{i}$ and with every iteration, the $y$-coordinate of the newly constructed point is decreased.

$(\Rightarrow)$ Given that $A_{n_{i}}$ is $\omega$-feasible with valid base $e_{i}$, we will show that the smaller angle between $n_{i}$ and all other outer normals is greater than or equal to $\pi / 2-\omega$ and that the set of all local minima with respect to $n_{i}$ is $e_{i}$.

Suppose there exists an outer normal $n_{j}$ such that $\angle n_{i} n_{j}<\pi / 2-\omega$. Let $p$ be a point in the interior of $e_{j}$. Since $e_{i}$ is an $\omega$-feasible base, there must exist a point $q$ such that $p$ is $\omega$-supported by $q$. However such a $q$ does not exist because of $n_{j}$.

Similarly, suppose there exists a local minimum point $p$ that is not contained in $\epsilon_{i}$. Again, the point $p$ is not $\omega$-supported.

Theorem 4.2 For fixed $\omega$, a polygon has a constant number of candidate edges that can be valid bases. These candidate edges can be obtained in $O(n)$ time.

Proof: Let $k=[2 \pi /(\pi / 2-\omega)]$. Cover the circle of directions with $k$ closed arcs, denoted by $a_{1}, a_{2}, \ldots a_{k}$, having the following property. The angle spanned by each of the arcs is exactly $(\pi / 2-\omega)$.

For edge $\boldsymbol{e}_{i}$, suppose that $n_{i}$ is contained in the open arc $a_{j}$. If edge $\epsilon_{i}$ is a valid base, then by Theorem 4.1 there are no other outer normals in the open arc $a_{j}$. If $n_{i}$ had been on the end of the closed arc $a_{j}$, then there can be at most one other normal on the other end of closed arc $a_{j}$. Therefore, each closed arc can contain the outer normal of at most 2 valid bases. Since there are $k$ arcs, there can be at most $2 k$ valid bases. But $k$ is a constant when $\omega$ is fixed; therefore, there are only a constant number of valid bases.

The algorithm for obtaining the valid bases follows from the discussion above. 
We now have all the tools needed to determine the $\omega$-feasibility of a simple polygon in linear time. A brief outline of the algorithm follows. The algorithm takes as input a simple polygon $A$ and parameter $\omega$.

\section{Algorithm: Determine the $\omega$-feasibility of a simple polygon.}

1. Let $B$ represent the set of candidate bases of $A$. There are only a constant number of edges in $B$ and they can be computed in linear time using the technique described in Theorem 4.2.

2. Test each edge $e_{i} \in B$ to see if it is valid in the following way.

- Check that the angle between normal $n_{i}$ and all other normals is no less than $\pi / 2-\omega$. This can be done in linear time.

- Verify that the set of all local minima with respect to $n_{i}$ is $e_{i}$. This can be done using the algorithm in Bose and Toussaint [5,6] which determines in linear time, given a polygon, a specified edge and a direction, whether the edge is the set of all local minima with respect to the given direction.

3. Output the valid bases

Testing an edge to see if it is valid takes linear time. However, since the number of edges tested is constant, step 2 is completed in linear time. The complexity of the algorithm is linear in the size of the input since the time to complete each step is at most linear. The correctness of the algorithm follows from Theorems 4.1 and 4.2 .

Theorem 4.3 The feasibility of a simple polygon in variable-angle stereolithography can be determined in $O(n)$ time.

Remark: The technique used to determine the feasibility of a simple polygon with $\omega=0$ provides an alternate linear time method to compute the feasibility in vertical stereolithography.

\subsection{Polyhedral Objects}

Similar to the two-dimensional case, with $\omega>0$, the base of an object no longer need be a face of the polyhedron (see Figure 7 ). However, we will assume that the base of an object is always a face of the polyhedron, since building an object on a vertex or an edge is unstable.

We say that a point $p$ in $A_{n(f)}$ is $\omega$-visible from a face $f$ if $p$ is above the plane $P(f)$ and there exists a polygonal path $\Pi$ from $p$ to $f$ such that $\Pi \in A_{n(f)}$ and the smaller angle between every pair of edges in $\Pi$ is no more than $\omega$. Thus, we observe the following.

Observation 4.3 A polyhedron $A_{n(f)}$ is $\omega$-feasible with valid base $f$ if and only if all points in $A_{n(f)}$ are $\omega$-visible from $f$.

We observe another property that is crucial to the development of a linear algorithm.

Observation 4.4 If a point $p$ is $\omega$-visible from $f$, then the path $\Pi$ from $p$ to $f$ is monotone with respect to direction $n(f)$. 
Theorem 4.4 A polyhedron $A_{n(f)}$ is $\omega$-feasible with valid base $f$ if and only if the angle between $n(f)$ and all other normals is no less than $\pi / 2-\omega$ and the set of all local minima with respect to $n(f)$ consists of face $f$.

\section{Proof:}

$(\Leftarrow)$ Given that all outer normals $n\left(f_{j}\right)$ distinct from $n(f)$ are such that the smaller angle between $\angle n\left(f_{j}\right) n(f) \geq \pi / 2-\omega$, and all local minima are contained in $f$, we will show that $A_{n(f)}$ is $\omega$-feasible with base $f$. We do this by exhibiting a construction such that every point in $A_{n(f)}$ is $\omega$-supported by the following construction.

Let $p \in A_{n(f)}$. Assume that $p \notin f$.

1. If $p$ is contained in $\operatorname{int}\left(A_{n(f)}\right)$, then let $q$ be the intersection point below $p$ and closest to $p$ of a vertical line through $p$ and $b d\left(A_{n(f)}\right)$.

2. If $p$ is contained in the interior of a face $f_{j}$, then let $q$ be a point on $f_{j}$ with lowest $y$-coordinate.

3. If $p$ is contained in the interior of an edge $e$, then let $q$ be a point with lowest $y$-coordinate in one of the two faces adjacent to $e$.

4. If $p$ is a vertex $v$, then let $q$ be a point with lowest $y$-coordinate in one of the faces adjacent to $v$.

In all cases, $q$ will have a lower $y$-coordinate than $p$ since $p$ is not a local minimum. By construction, the smaller angle between $\overrightarrow{p q}$ and $n(f)$ is no more than $\omega$. Therefore, $p$ is $\omega$-supported. If $q \in f$, then we are done. If $q \notin f$, we must show that $q$ is $\omega$-supported. This can be done by repeating steps $1,2,3,4$ with $q$. The construction must end with a point on $f$ since $f$ contains all local minima with respect to $n(f)$ and with every iteration, the $y$-coordinate of the newly constructed point is decreased.

$(\Rightarrow)$ Given that $A_{n(f)}$ is $\omega$-feasible with valid base $f$, we will show that the smaller angle between $n(f)$ and all other outer normals is greater than or equal to $\pi / 2-\omega$ and that the set of all local minima with respect to $n(f)$ is $e_{i}$.

Suppose there exists an outer normal $n\left(f_{j}\right)$ such that $\angle n\left(f_{j}\right) n(f)<\pi / 2-\omega$. Let $p$ be a point in the interior of $f_{j}$. Since $f$ is an $\omega$-feasible base, there must exist a point $q$ such that $p$ is $\omega$-supported by $q$. However such a $q$ does not exist because of $n\left(f_{j}\right)$.

Similarly, suppose there exists a local minimum point $p$ that is not contained in $f$. Again, the point $p$ is not $\omega$-supported.

Theorem 4.5 For fixed $\omega$, a polyhedron has a constant number of candidate faces that can be valid bases. These faces can be obtained in $O(n)$ time.

Proof: Let us consider the spherical coordinates $(\phi, \rho)$ of the sphere of directions $\mathcal{S}$ centered at the origin where the angle $\phi$ is in the set $[0,2 \pi)$ and the angle $\rho$ is in the interval $[-\pi / 2, \pi / 2]$. We first divide the sphere of directions into $k=\lceil\pi /(\pi / 4-\omega / 2)\rceil$ slices with parallel circles in the 


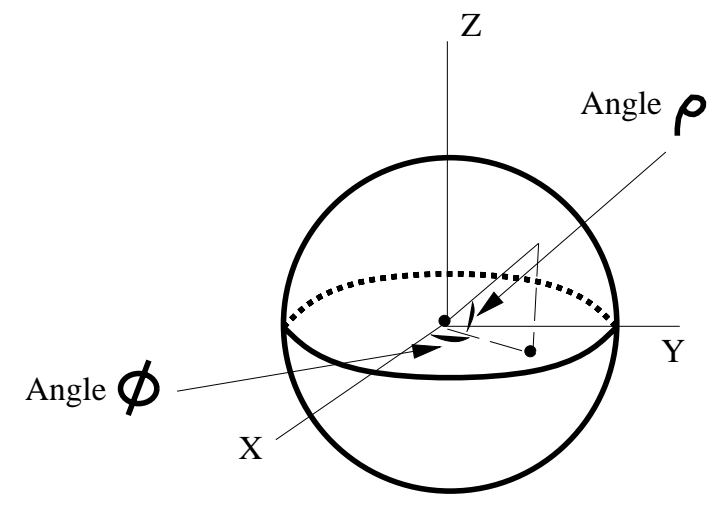

Figure 8: Spherical Coordinates.

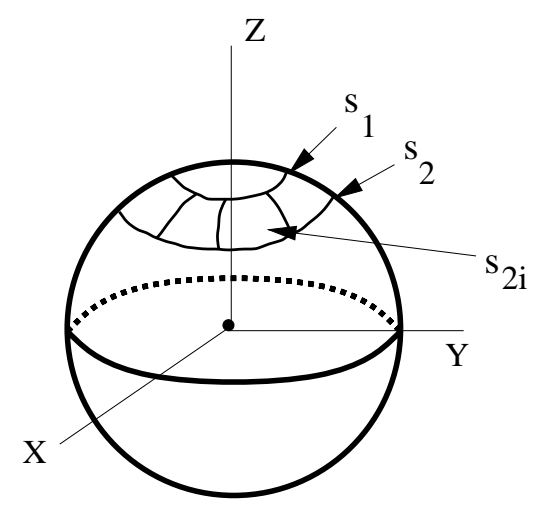

Figure 9: Slices and pieces of the sphere of directions.

following way. Slice $s_{1}$ contains all points where $\rho \in[\pi / 2, \pi / 2-(\pi / 4-\omega / 2)]$. Slice $s_{2}$ contains all points where $\rho \in[\pi / 2-(\pi / 4-\omega / 2), \pi / 2-2(\pi / 4-\omega / 2)]$. Slice $s_{i}$ contains all points where $\rho \in[\pi / 2-(i-1)(\pi / 4-\omega / 2), \pi / 2-i(\pi / 4-\omega / 2)]$. See Figure 9.

Each slice $s_{i}$ is further subdivided into $m=\lceil 2 \pi /(\pi / 4-\omega / 2)\rceil$ pieces in the following way. Piece $s_{i 1}$ contains all points where $\phi \in[0, \pi / 4-\omega / 2]$ and $\rho \in[\pi / 2-(i-1)(\pi / 4-\omega / 2), \pi / 2-i(\pi / 4-\omega / 2)]$. Piece $s_{i 2}$ contains all points where $\phi \in[\pi / 4-\omega / 2,2(\pi / 4-\omega / 2)]$ and $\rho \in[\pi / 2-(i-1)(\pi / 4-$ $\omega / 2), \pi / 2-i(\pi / 4-\omega / 2)]$. Piece $s_{i j}$ contains all points where $\phi \in[(j-1)(\pi / 4-\omega / 2), j(\pi / 4-\omega / 2)]$ and $\rho \in[\pi / 2-(i-1)(\pi / 4-\omega / 2), \pi / 2-i(\pi / 4-\omega / 2)]$.

By construction, any pair of points in a piece $s_{i j}$, represents a pair of directions $d_{1}$ and $d_{2}$ such that the smaller angle between $d_{1}$ and $d_{2}$ is strictly less than $\pi / 2-\omega$. Therefore, the outer normals of two feasible bases cannot lie in the same piece.

There are $\mathrm{km}$ pieces. Notice that $\mathrm{km}$ no more than $\pi^{2} /(\pi / 2-\omega)^{2}$. Since $\omega$ is fixed, $k m \in O(1)$. Each piece can contain at most 1 feasible base. Therefore, there are $O(1)$ feasible bases. 
We now have all the tools needed to determine the feasibility of a simple polyhedron in linear time. A brief outline of the algorithm follows. The algorithm takes as input a simple polyhedron $A$ and parameter $\omega$.

\section{Algorithm: Determine the $\omega$-feasibility of a simple polyhedron.}

1. Let $B$ represent the set of candidate bases of $A$. There are only a constant number of faces in $B$ and they can be computed in linear time using the technique described in the proof of Theorem 4.5.

2. Test each face $f_{i} \in B$ to see if it is valid in the following way.

- Check that the angle between normal $n\left(f_{i}\right)$ and all other normals is no less than $\pi / 2-\omega$. This can be done in linear time.

- Verify that the set of all local minima with respect to $n\left(f_{i}\right)$ is $f_{i}$. This can be done using the algorithm in Bose, van Kreveld and Toussaint [7], which determines in linear time, given a polyhedron, a face and a direction, whether the face is the set of all local minima with respect to the given direction.

3. Output the valid bases

Testing a face to see if it is valid takes linear time. However, since the number of faces tested is constant, step 2 is completed in linear time. The complexity of the algorithm is linear in the size of the input since the time to complete each step is at most linear. The correctness of the algorithm follows from Theorem 4.4, 4.5.

Theorem 4.6 The feasibility of a simple polyhedron in variable-angle stereolithography can be determined in $O(n)$ time.

Remark: The technique used to determine the feasibility of a simple polyhedron with $\omega=0$ provides an alternate linear time method to compute the feasibility in standard stereolithography.

The initial assumption that the base of an object is always a face of the given polyhedron may be slightly weakened at the cost of a $\log n$ factor. One might argue that although the construction of an object from a vertex or edge may be unstable, it is reasonable to assume that the object is placed on a face of the convex hull of the object. After the construction of the convex hull of the object, we see that determining its feasibility under this weaker assumption can be done in linear time from the discussion above. Therefore, we have the following.

Theorem 4.7 Given a simple polyhedron $A$, if the base of $A$ can be a face of its convex hull, then feasibility in variable-angle stereolithography can be determined in $O(n \log n)$ time.

\section{$5 \quad$ Relation to Gravity Casting}

A well-known technique used in the manufacturing of goods is gravity casting. A mold, as defined in [9], refers to the whole assembly of parts that make up a cavity into which liquid is poured to 
give the shape of the desired component when the liquid hardens. Given a mold (modelled as a polyhedron), establishing whether there exists an orientation that allows the filling of the mold using only one pin gate (the pin gate is the point from which the liquid is poured into the mold) as well as determining an orientation that allows the most complete fill are two major problems in the field of gravity casting. These and other related problems in molding have been studied by Bose and Toussaint [5], [6], Bose, Van Kreveld and Toussaint [7], Fekete and Mitchell [13], and Rosenbloom and Rappaport [23] from an algorithmic and geometric point of view.

In [7], it is shown that given an arbitrary polyhedron on $n$ vertices, in $O\left(n^{2}\right)$ time all the orientations that allow the polyhedron to be filled from one pin gate can be found. It is also shown in [7] that this problem is $n^{2}$-hard [14], which leads one to believe that improving this time complexity will be difficult. However, for the restricted case of a polyhedron that can be built using variable-angle stereolithography, we have the following theorem relating the two manufacturing processes.

Theorem 5.1 A polyhedral object formed by variable-angle stereolithography can be formed by gravity casting using only one pin gate.

Proof: In [7], it is shown that an object can be formed by gravity casting using only one pin gate if and only if the pin gate is the only local maximum. The theorem follows from Theorem 4.4

\section{Relation to Polyhedral Terrains}

Polyhedral terrains are important structures in geographic information systems ([16], [15]) and computational geometry ([11], [8]). A polyhedral terrain is defined as follows.

A terrain $T$ is a two-dimensional triangulated polyhedral surface with $n$ vertices $V=\left\{v_{1}, v_{2}, \ldots, v_{n}\right\}$. Each vertex $v_{i}$ is specified by three real numbers $\left(x_{i}, y_{i}, z_{i}\right)$ which are its cartesian coordinates and $z_{i}$ is referred to as the height of vertex $v_{i}$. It is convenient to assume that $z_{i}$ is non-negative so that if the X-Y plane is associated with sea-level, no points on the terrain are below sea-level. Let $P=\left\{p_{1}, p_{2}, \ldots, p_{n}\right\}$ denote the orthogonal projections of the points $V=\left\{v_{1}, v_{2}, \ldots, v_{n}\right\}$ on the $\mathrm{X}-\mathrm{Y}$ plane, i.e., each point $p_{i}$ is specified by the two real numbers $\left(x_{i}, y_{i}\right)$. It is assumed that the set $P=\left\{p_{1}, p_{2}, \ldots, p_{n}\right\}$ is in general position, i.e., no three points are collinear and no four are cocircular so that the projections of the edges of the polyhedral surface onto the X-Y plane determine a triangulation of $P$ (hence the term triangulated polyhedral surface). We refer to the triangulation as the underlying triangulated planar graph associated with the terrain. Therefore we can view a terrain $\mathrm{T}$ as the graph of a polyhedral function $z=F(x, y)$, defined over the convex hull of $P$. Sometimes a polyhedral terrain is assumed to be a monotone polyhedral surface, i.e., a polyhedral surface having exactly one intersection with every vertical line [11]. In our case we assume the stronger condition that the intersection of every vertical line in the interior of the convex hull of $P$ with the polyhedral surface is a single point. Intuitively, a monotone terrain admits vertical walls whereas our definition does not. Since the orthogonal projection of T onto the X-Y plane is a planar straight-line subdivision or map, it follows that $\mathrm{T}$ has $O(n)$ edges and $O(n)$ triangular faces. A polyhedron $P$ is a solid terrain provided that it can be positioned such that it is vertically visible from one of its faces. 
We have the following theorem.

Theorem 6.1 Determining if a polyhedron $P$ is a solid terrain can be achieved in $O(n)$ time.

In fact, the polyhedra that can be formed by variable-angle stereolithography provide a generalization of the standard polyhedral terrains studied in geographic information systems. Our algorithms recognize whether a polyhedral surface is a terrain that allows overhangs, a more realistic terrain.

\section{$7 \quad$ Relation to Clamping}

Grasping is a well known research area in robotics. Much research has been done on the problem of gripping or immobilizing an object with a multifingered hand [2, 12, 19, 20, 24]. Recently, researchers have considered the problem of finding a "geometric" grip of a planar object [25, 10 , 1, 26]. Souvaine and Van Wyk [25] studied the problem of clamping a polygon with a pair of parallel line segments, motivated by robot hands known as parallel jaw grippers that are pairs of parallel plates. Each plate is referred to as a gripper. Informally, a polygon $P$ is clamped in the plane when it is "securely" held between the two grippers (modelled in the plane by a pair of line segments forming the opposite sides of a rectangle) such that $P$ does not rotate or slip out of the gripper when the gripper is squeezed. A polygon is called clampable if there exists a clamp for every positive length gripper. Bose, Bremner and Toussaint [4] studied the three dimensional problem of clamping polyhedron with a pair of parallel rectangles, representing the parallel jaw grippers. They show that a polygonal or polyhedral object built with vertical stereolithography is clampable.

\section{Conclusions}

We have presented linear time algorithms for determining whether or not an object (modelled as a polygon or polyhedron) can be manufactured using vertical stereolithography. For feasible objects our algorithms report a description of all the orientations in which the object can be made. We also determine feasibility of both polygonal and polyhedral objects constructed using variable-angle stereolithography. We give an $O(n)$ time algorithm for polygons and an $O(n \log n)$ as well as an $O(n)$ time algorithm for polyhedra. We show that objects formed using variable-angle stereolithography can also be constructed using another manufacturing process known as gravity casting. We also show that the polyhedral objects formed by stereolithography are closely related to polyhedral terrains, and introduce a new more general and realistic class of polyhedral terrains. Finally, we show that polygonal and polyhedral objects built by stereolithography can be clamped with parallel jaw grippers.

This initial investigation provides many directions for further research. For example, in the variable-angle stereolithography model, we assumed that an object cannot be built on a vertex or edge since the object would not be stable. However, in practice, objects may be constructed on a vertex or edge by introducing support stilts as the object is being built in order to maintain stability. It would be interesting to incorporate this into the variable-angle model. 


\section{References}

[1] Albertson, M., R. Haas, J. O'Rourke, Some results on clamping a polygon, Tech. Rep. No. 003, Department of Computer Science, Smith College, 1990.

[2] Baker, B., S. Fortune, and E. Grosse, Stable prehension with a multi-fingered hand: extended abstract, Proc. IEEE Intl. Conf. on Robotics and Automation, St. Louis, Missouri, 1985.

[3] Bondy, J. and U.S.R. Murty, Graph theory with applications. Elsevier Science, New York, New York, 1976.

[4] Bose, P., D. Bremner, and G. Toussaint, All convex polyhedra can be clamped with parallel jaw grippers, Technical Report, School of Computer Science, McGill University, to appear.

[5] Bose, P. and G. Toussaint, Geometric and computational aspects of gravity casting, to appear in C.A.D.

[6] Bose, P. and G. Toussaint, Geometric and computational aspects of injection molding. Proc. Third International Conf. on CAD and Computer Graphics, August 1993, Beijing, China, p. 237-242, also available as Technical Report No. SOCS 92.16, School of Computer Science, McGill University, 1992.

[7] Bose, P., M. Van Kreveld and G. Toussaint, Filling polyhedral molds, Proc. Third WADS (1993), Lecture Notes in Computer Science 709, Springer-Verlag, p. 210-221, also available as Technical Report No. SOCS 93.1, School of Computer Science, McGill University, 1993.

[8] Bose, P., T. Shermer, G. Toussaint, and B. Zhu, Guarding polyhedral terrains, Proc. Allerton Conf., Urbana-Champaign, Ill., October 1992.

[9] Bown, J., Injection moulding of plastic components. McGraw-Hill, England, 1979.

[10] Capoyleas, V., Clamping of polygonal objects. Pattern Recognition Letters, 14, pp. 704-714, 1993.

[11] Cole, R. and M. Sharir, Visibility problems for polyhedral terrains. Journal of Symbolic Computation, 7, p.11-30, 1989.

[12] Czyzowicz, C., I. Stojmenovic and J. Urrutia, Immobilizing a shape in the plane, Proc. 3rd Canad. Conf. Comput. Geom., Vancouver, B.C., pp. 41-45, 1991.

[13] Fekete, S. and J. Mitchell, Geometric aspects of injection molding. State University of New York, Stony Brook, Manuscript, 1993.

[14] Gajentaan, A. and M.H. Overmars, $O\left(n^{2}\right)$ difficult problems in computational geometry. Technical Report, Dept of Computer Science, Utrecht University, 1993.

[15] Goodchild, M. and J. Lee, Coverage problems in visibility regions on topological surfaces. Annals of Operations Research, 18, p.175-186, 1989.

[16] Lee, J., Analyses of visibility sites on topographic surfaces. Int. J. Geographic Information Systems, 5,4, p.413-429, 1991. 
[17] McCallum, D. and D. Avis, A linear algorithm for finding the convex hull of a simple polygon, Information Processing Letters, 9, p.201-206, 1979.

[18] Melkman, A., On-line construction of the convex hull of a simple polyline. Information Processing Letters, 25, p.11-12, 1987.

[19] Mishra, B., J. Schwartz, and M. Sharir, On the existence and synthesis of multifinger positive grips, Algorithmica, 2, pp. 541-588, 1987.

[20] Montejano, L., and J. Urrutia, Immobilizing figures on the plane, Proc. 3rd Canad. Conf. Comput. Geom., Vancouver, B.C., pp. 46-49, 1991.

[21] Preparata, F. and S. Hong, Convex hulls of finite sets of points in two and three dimensions, Communications of the ACM, 2,20, p. 87-93, 1977.

[22] Preparata, F. and M. Shamos, Computational geometry: an introduction, Springer-Verlag, New York, New York, 1985.

[23] Rosenbloom, A. and R. Rappaport, Moldable and castable polygons, Proc. of the Fourth Canadian Conference on Computational Geometry, St. John's, Newfoundland, p. 322- 327, 1992.

[24] Salisbury, J., Design and control of an articulated hand, Proc. 1st Intl. Symp. on Design and Synthesis, Tokyo, Japan, 1984.

[25] Souvaine, D., and C. Van Wyk, Clamping a polygon. to appear in The Visual Computer, 1994, also available as Tech. Rep. No. LCSR-TR-98, Department of Computer Science, Rutgers University, 1989.

[26] Teichmann, M., and B. Mishra, Reactive algorithms for grasping using a modified parallel jaw gripper, to appear in Proc. IEEE Intl. Conf. on Robotics and Automation. May 1994.

[27] Watson, D. and M. Yeung, Geometric modelling from CT scans for stereolithography. Proc. Third International Conf. on CAD and Computer Graphics, August 1993, Beijing, China, p. 417-422. 